\section{Der „Klassiker" - konkurrenzlos.}

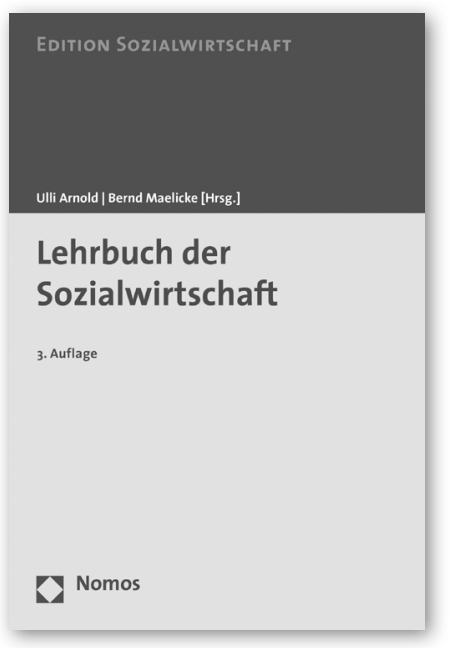

\section{Lehrbuch der \\ Sozialwirtschaft}

Von Prof. Dr. Dr. h.c. Ulli Arnold, Universität Stuttgart und Prof. Dr. Bernd Maelicke, Leuphana-Universität Lüneburg, Deutsches Institut für Sozialwirtschaft, Lüneburg

3. Auflage 2008, ca. $600 \mathrm{~S}$.,

brosch., ca. 39,- $€$,

ISBN 978-3-8329-2680-9

(Edition Sozialwirtschaft, Bd. 9)

Erscheint Februar 2008

Die rapide Veränderung der politischen und strukturellen Rahmenbedingungen für die Sozialwirtschaft erfordert grundlegende Änderungen in der Ausbildung und Weiterbildung von Führungskräften sozialer Dienstleistungsorganisationen. Die vollständig aktualisierte und neu bearbeitete 3. Auflage vermittelt Basiswissen und aktuelle Informationen zu den wichtigsten Themen.

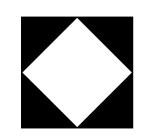

Nomos

Bitte bestellen Sie bei Ihrer Buchhandlung oder bei Nomos | Telefon 07221/2104-37 | Fax -43 | www.nomos.de | sabine.horn@nomos.de

\title{
Rechtsformen für Sozialunternehmen
}

\author{
Ulla Engler
}

Die im letzten Jahr in Kraft getretene Reform des Genossenschaftsrechts hat die Palette praktikabler Rechtsformen für soziale Organisationen um das Modell "Sozialgenossenschaft " erweitert. Eine aktuelle Übersicht vergleicht die wichtigsten rechtlichen Optionen für soziale Organisationen.

Für soziale Organisationen, die sich in der Gründungsphase befinden und die den Gemeinnützigkeitsstatus aus (steuerlichen) Gründen erlangen möchten, stellt sich die Frage der geeigneten Rechtsform. Aber auch bereits seit Längerem tätige eingetragene Vereine oder gemeinnützige GmbHs unterziehen oftmals ihre Rechtsformwahl nach einigen Jahren einer Überprüfung. Ein Grund hierfür kann sein, dass die wirtschaftliche Betätigung der Organisation zugenommen hat oder Haftungsrisiken verringert werden sollen.

Zur Disposition stehen für soziale Organisationen in erster Linie der eingetragene Verein (e. V.), die gemeinnützige $\mathrm{GmbH}$ (gGmbH), die Gesellschaft bürgerlichen Rechts (GbR bzw. BGB-Gesellschaft) und die eingetragene Genossenschaft (e. G.). Insbesondere letztere hat mit dem »Gesetz zur Einführung der Europäischen Genossenschaft und zur Änderung des Genossenschaftsrechts«, das im letzten Jahr in Kraft getreten ist, an Bedeutung gewonnen (vgl. SOZIALwirtschaft 6/2006). Ein Ziel dieser Reform war die Erleichterung der Gründung von gemeinnützigen Genossenschaften: In der Vergangenheit regelte das Genossenschaftsgesetz, dass eine Genossenschaft dem »Erwerb und der Wirtschaft « ihrer Mitglieder dient. Diese Formulierung führte oftmals dazu, dass Finanzverwaltungen Genossenschaften die Anerkennung der Gemeinnützigkeit mit

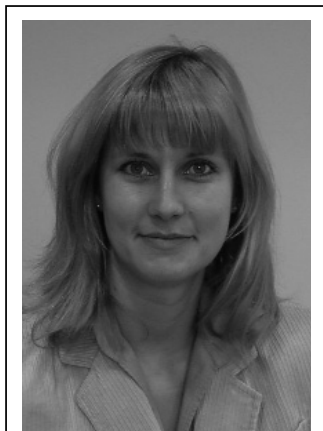

Die

Rechtsanwältin Ulla Engler ist Referentin für Organisationsrecht beim Gesamt-

verband des Paritätischen Wohlfahrtsverbandes in Berlin. Im Informationsdienst SOZIALwirtschaft aktuell gibt sie regelmäßig Auskunft zu aktuellen Fragen des Vereinsrechts. E-Mail organisationsrecht@ paritaet.org

der Begründung verweigerten, gemeinnützige Körperschaften hätten nach der Abgabenordnung die »Allgemeinheit « zu fördern und nicht nur ihre Mitglieder. Die Erweiterung des Förderzwecks in $\S 1$ des neuen Genossenschaftsgesetzes öffnet nunmehr die Rechtsform der Genossenschaft auch für »soziale oder kulturelle Belange«.

Welche Rechtsform im konkreten Einzelfall für eine soziale Organisation geeignet ist, hängt von vielen Faktoren $\mathrm{ab}$ und muss sorgfältig abgewogen werden. $\mathrm{Zu}$ berücksichtigen ist beispielsweise neben der Anzahl der erforderlichen Gründungsmitglieder das notwendige Mindestkapital, die Haftung für Verbindlichkeiten oder die Möglichkeit, den Gemeinnützigkeitsstatus erlangen zu können.

Die nebenstehende Tabelle kann einen aktuellen Überblick über wichtige Rechtsformen und geben und als Entscheidungshilfe dienen. 
Vergleich der Rechtsformen für soziale Organisationen

\begin{tabular}{|c|c|c|c|c|}
\hline & $\begin{array}{l}\text { eingetragene } \\
\text { Genossenschaft } \\
\text { (e. G.) } \\
\text { (Sozialgenossen- } \\
\text { schaften) }\end{array}$ & $\begin{array}{l}\text { eingetragener } \\
\text { Verein } \\
\text { (e. V.) }\end{array}$ & $\begin{array}{l}\text { Gesellschaft mit be- } \\
\text { schränkter Haftung } \\
(\mathrm{GmbH})\end{array}$ & $\begin{array}{l}\text { Gesellschaft } \\
\text { bürgerlichen Rechts } \\
\text { (GbR bzw. } \\
\text { BGB-Gesellschaft) }\end{array}$ \\
\hline Rechtsgrundlage & $\begin{array}{l}\text { Genossenschafts- } \\
\text { gesetz (GenG }\end{array}$ & $\S \S 21 \mathrm{ff} . \mathrm{BGB}$ & $\begin{array}{l}\text { GmbH-Gesetz } \\
(\mathrm{GmbH})\end{array}$ & $\S \S 705$ ff. BGB \\
\hline $\begin{array}{l}\text { Anzahl der Grün- } \\
\text { dungsmitglieder }\end{array}$ & $\begin{array}{l}3 \text { Personen } \\
\text { (\$ } 4 \text { GenG) }\end{array}$ & $\begin{array}{l}7 \text { Personen } \\
(\S 56 \mathrm{BGB})\end{array}$ & $\begin{array}{l}1 \text { Person } \\
(\S 1 \mathrm{GmbHG})\end{array}$ & 2 Personen \\
\hline Zweck & $\begin{array}{l}\text { Förderung des Er- } \\
\text { werbs oder der Wirt- } \\
\text { schaft der Mitglieder } \\
\text { ( } § 1 \text { GenG) } \\
\text { soziale oder kulturel- } \\
\text { le Belange } \\
(\S 1 \text { GenG) }\end{array}$ & $\begin{array}{l}\text { nicht auf einen wirt- } \\
\text { schaftlichen Ge- } \\
\text { schäftsbetrieb gerich- } \\
\text { tet (\$ } 21 \mathrm{BGB})\end{array}$ & $\begin{array}{l}\text { jeder gesetzlich } \\
\text { zulässige Zweck } \\
\text { (§ } 1 \mathrm{GmbHG)}\end{array}$ & $\begin{array}{l}\text { gemeinsamer Zweck } \\
(\S 705 \text { BGB) }\end{array}$ \\
\hline Mindestkapital & $\begin{array}{l}\text { nicht erforderlich, } \\
\text { kann jedoch in der } \\
\text { Satzung festgelegt } \\
\text { werden (§ 8a GenG) }\end{array}$ & nicht erforderlich & $\begin{array}{l}\text { 25.000 Euro } \\
\text { (§ } 5 \text { GmbHG) } \\
\text { (soll auf } 10.000 \text { Euro } \\
\text { abgesenkt werden) }\end{array}$ & nicht erforderlich \\
\hline $\begin{array}{l}\text { Haftung für } \\
\text { Verbindlichkeiten }\end{array}$ & $\begin{array}{l}\text { Haftung beschränkt } \\
\text { auf Vermögen der } \\
\text { Genossenschaft } \\
\text { (§ } 2 \text { GenG) }\end{array}$ & $\begin{array}{l}\text { Haftung beschränkt } \\
\text { auf Vermögen des } \\
\text { Vereins }\end{array}$ & $\begin{array}{l}\text { Haftung beschränkt } \\
\text { auf Vermögen der } \\
\text { Gesellschaft } \\
\text { (§ } 13 \text { Abs. } 2 \text { GmbHG) }\end{array}$ & $\begin{array}{l}\text { persönliche und ge- } \\
\text { meinsame Haftung } \\
\text { aller Gesellschafter }\end{array}$ \\
\hline $\begin{array}{l}\text { Gemeinnützig- } \\
\text { keitsstatus } \\
\text { (Befreiung von der } \\
\text { Körperschaftssteuer } \\
\text { und der Gewerbe- } \\
\text { steuer) }\end{array}$ & $\begin{array}{l}\text { Befreiung gemäß } § 5 \\
\text { Abs. } 1 \text { Nr. } 9 \text { KStG } \\
\S 3 \text { Nr. } 6 \text { GewStG }\end{array}$ & $\begin{array}{l}\text { Befreiung gemäß } \\
\text { § } 5 \text { Abs. } 1 \text { Nr. } 9 \text { KStG } \\
\text { § } 3 \text { Nr. } 6 \text { GewStG }\end{array}$ & $\begin{array}{l}\text { Befreiung gemäß } \\
\text { § } 5 \text { Abs. } 1 \text { Nr. } 9 \text { KStG } \\
\text { § } 3 \text { Nr. } 6 \text { GewStG }\end{array}$ & Keine Befreiung \\
\hline Organe & $\begin{array}{l}\text { Vorstand (§ } 9 \text { GenG) } \\
\text { Aufsichtsrat (\$ } 9 \\
\text { GenG) } \\
\text { Generalversammlung } \\
\text { (§ } 43 \text { GenG) }\end{array}$ & $\begin{array}{l}\text { Vorstand (§ } 26 \text { BGB) } \\
\text { Mitgliederversamm- } \\
\text { lung ( } \$ 32 \text { BGB) }\end{array}$ & $\begin{array}{l}\text { Geschäftsführer } \\
\text { (§ } 35 \text { GmbHG) } \\
\text { Gesellschafterver- } \\
\text { sammlung } \\
\text { (§ } 48 \text { GmbHG) }\end{array}$ & $\begin{array}{l}\text { Gesellschafter } \\
\text { Geschäftsführer }\end{array}$ \\
\hline Eintragung & $\begin{array}{l}\text { Genossenschaftsregi- } \\
\text { ster ( } \$ 10 \text { GenG) }\end{array}$ & $\begin{array}{l}\text { Vereinsregister } \\
(\S 21 \mathrm{BGB})\end{array}$ & $\begin{array}{l}\text { Handelsregister } \\
(\$ 10 \mathrm{GmbHG})\end{array}$ & Nicht erforderlich \\
\hline Besonderheiten & $\begin{array}{l}\text { Pflichtprüfung zur } \\
\text { Feststellung der wirt- } \\
\text { schaftlichen Verhält- } \\
\text { nisse und der Ord- } \\
\text { nungsmäßigkeit der } \\
\text { Geschäftsführung } \\
\text { (§ } 53 \text { GenG) } \\
\text { Pflichtmitgliedschaft } \\
\text { in einem prüfungs- } \\
\text { verband (\$ } 54 \text { GenG) }\end{array}$ & & & $\begin{array}{l}\text { Keine juristische per- } \\
\text { son des privaten } \\
\text { Rechts }\end{array}$ \\
\hline
\end{tabular}

